\title{
Antenatal diagnosis and outcome of agenesis of corpus callosum: A retrospective review of 33 cases
}

\author{
Özgür Özyüncü, Aslıhan Yazıcıŏ̆lu, Mert Turğal \\ Department of Obstetrics and Gynecology, Hacettepe University Faculty of Medicine, Ankara, Turkey
}

\section{Abstract}

Objective: To present antenatal sonographic findings and postnatal outcome of a population of foetuses diagnosed with agenesis of corpus callosum.

Material and Methods: The database of our ultrasound laboratory was retrospectively searched for cases of agenesis of the corpus callosum suspected at antenatal sonography between 2002 and 2012. The following variables were assessed: maternal age, gestational age at diagnosis, gender, any additional cerebral and extra-cerebral malformations, results of karyotype analysis and pregnancy and foetal/neonatal outcomes.

Results: During the study period, 33 foetuses with agenesis of the corpus callosum were identified antenatally, with a male preponderance. The mean maternal age was 28.48 years. In all cases, pre/postnatal MRI and/or necropsy were performed in order to confirm the diagnosis. Among those, there were additional brain findings in 23 (69.7\%) and additional extra-cerebral anomalies in 3 (9.1\%) foetuses. Karyotype analysis was performed in 21 of $33(63.6 \%)$ cases. As for pregnancy outcome, the pregnancy was terminated in 14 (42.4\%) of the remaining 19 foetuses; eighteen (54.5\%) were delivered near term and one (3.1\%) who was delivered prematurely died during the neonatal period.

Conclusion: The diagnosis of congenital brain malformation is a challenging issue, since additional findings have a considerable effect on prognosis; detailed examination with genetic counselling should be performed.

(J Turk Ger Gynecol Assoc 2014; 15: 18-21)

Key words: Agenesis of corpus callosum, foetal MRI, prenatal diagnosis, ultrasound

Received: 28 September, $2013 \quad$ Accepted: 14 November, 2013

\section{Introduction}

The corpus callosum (CC) is the main junction between the two cerebral hemispheres, comprised of approximately 180 million axons, extending from the frontal lobe anteriorly to above the quadrigeminal plate posteriorly (1). Functionally, CC permits not only the information transfer between cerebral hemispheres, but also inhibition of concurrent activity in the contralateral hemisphere (1). From anterior to posterior it has 4 parts, namely the rostrum, genu, body and splenium (1). The anterior portion of the genu forms at the same time as the posterior callosal body, after which the splenium forms, and the last part to develop is the rostrum (1). By the end of the 20th week of gestation, the $\mathrm{CC}$ is well established with a shape similar to that of the adult, but with less myelination, reaching adult size by the age of two (1). The prevalence of agenesis of corpus callosum (ACC) varies in different studies, depending on the population studied and the diagnostic criteria, between $0.3 \%$ and $0.7 \%$ in the general population and $2 \%$ and $3 \%$ in the developmentally disabled population (2). Sonographic findings of ACC were first described by Comstock et al. (3), divided into direct findings such as the complete or partial absence of $\mathrm{CC}$ in the midsagittal plane, and indirect findings, such as colpocephaly, obliteration of the cavum septum pellucidum, elevation and dilatation of the 3rd ventricle and abnormal course of the pericallosal artery (4). Congenital anomalies of CC are commonly associated with other cerebral and extra-cerebral malformations, aneuploidies, genetic syndromes or inborn errors of metabolism, but can also be found in asymptomatic individuals with normal intelligence (5).

By the help of advanced foetal imaging techniques, antenatal diagnosis of complete callosal agenesis is feasible with midtrimester sonography. Beside this, accurate diagnosis of ACC and differentiation between partial and complete forms is still a challenge. Due to the uncertainty of postnatal outcome, giving a prognosis is extremely difficult. Our aim in this study is to report the results of follow-up and perinatal outcomes of cases with ACC between 2002 and 2012.

\section{Material and Methods}

Data were collected from our Maternal-Fetal Medicine Unit database. A retrospective database search for ACC was performed and all of the cases with a diagnosis of ACC were selected between 2002 and 2012. The informed consent of 
each participant was taken. The statistical analyses were performed using the Statistical Package for the Social Sciences (SPSS) 17.0 (SPSS Inc., Chicago, IL, USA). For each case, the following variables were assessed: maternal age, gestational age at diagnosis time, foetal gender, additional cerebral and extra-cerebral malformations, karyotype analysis, pregnancy and foetal/neonatal outcome. The diagnoses were confirmed at follow-up by pathology or postnatal neuroimaging with ultrasonography and magnetic resonance imaging (MRI).

Ultrasound investigations were carried out using Siemens Sonoline Antares (Siemens AG, North Rhine-Westphalia, Germany) and Voluson 730 pro (GE Healthcare, Milwaukee, WI, USA).

\section{Results}

During the study period, 33 foetuses with ACC were identified antenatally; the general features are given in Table 1 . The mean age of the pregnant women was $28.48 \pm 5.60$ years. The mean gravida was $2.21 \pm 1.38$ and $39.39 \%$ of the patients were primipara. The mean gestational age at initial diagnosis was $24.06 \pm 4.50$ weeks. Twenty-four of the 33 foetuses (72.7\%) were male and $9(27.3 \%)$ were female.

Postnatal sonography and MRI were available in 14 cases (42.4\%) and autopsy reports were available in ten of the fourteen cases in which a post-mortem examination had been performed (71.4\%).

Among the 33 foetuses with ACC, seven (21.2\%) showed isolated ACC. Additional findings were given in Table 1. Following sonographic diagnosis, foetal brain MRI was performed in four cases and confirmed the ultrasound findings in all of them. Additional cerebral findings were encountered in 23 foetuses (69.7\%). Additional extra-cerebral anomalies were recognised in three foetuses $(9.1 \%)$, including cerebro-oculo-fascio-skeletal syndrome, multicystic dysplastic kidney and single umbilical artery with a single kidney.

Eighteen of 33 foetuses had complete ACC (54.5\%). Thirteen of these cases had additional cerebral findings (72.2\%), 1 had multicystic dysplastic kidney (5.5\%) and the remaining 4 were isolated cases of complete ACC (22.3\%). Four out of 18 cases had chromosomal abnormalities (22.3\%), including mosaic trisomy 8 , ring chromosome 14 , trisomy 18 and the partial duplication of $6 \mathrm{p}$. All of these chromosomal abnormalities were found in complete ACC cases presenting with additional cerebral findings. Ten out of 18 cases were terminated (55.6\%); the remaining 8 were alive in the neonatal period (44.4\%).

Fifteen of 33 foetuses had partial ACC (45.5\%). Ten of these cases had additional cerebral findings (66.7\%), 2 presented with cerebro-oculo-fascio-skeletal syndrome and single umbilical artery with single kidney (13.3\%) and the remaining 3 were isolated cases of partial ACC (20.0\%). Two out of 15 cases had chromosomal abnormalities (13.3\%), one case each of trisomy 21 and trisomy 18. Both of these chromosomal abnormalities were found in partial ACC cases presenting with additional cerebral findings. Four out of 15 cases were terminated (26.6\%), 10 were alive in the neonatal period (66.7\%) and one $(6.7 \%)$ who was delivered prematurely at 32 weeks, died during the neonatal period because of necrotising enterocolitis. The details are given in Table 1.

Twelve out of 33 (36.4\%) cases did not undergo karyotype analysis due to the late admission in 6 (50.0\%), and patient preference in the other 6 (50.0\%). Postnatal karyotype analysis was not performed on the basis of the initial postnatal evaluation. Three cases presenting with additional extra-cerebral findings, as mentioned before, had normal karyotype analysis results.

\section{Discussion}

Agenesis of corpus callosum is one of the most common central nervous system (CNS) abnormalities diagnosed in the antenatal period (4). ACC can present in different forms, complete or partial, isolated or associated with other cerebral and extra-cerebral abnormalities. In the literature, it has been recommended to evaluate the development of corpus callosum between the $20^{\text {th }}$ and $24^{\text {th }}$ gestational weeks since it is difficult to estimate the presence of callosal abnormalities before the $18^{\text {th }}$ gestational week. In accordance with the literature, we diagnosed ACC with a mean gestational age of 24 weeks.

Vasudevan et al. (1) reported that isolated ACC accounts for approximately $50 \%$ of patients diagnosed with ACC. In the other half, various CNS abnormalities were described (1). In our study, we found that 7 out of $33(21.2 \%)$ had isolated ACC. On the other hand, Mangione et al. (6) reported a large series of 175 patients in which CNS abnormalities were found in $50 \%$ of isolated ACC cases. It has also been shown that the presence of extra-cerebral abnormalities occurs in about $65 \%$ of antenatally diagnosed ACC patients (7). Consistent with the literature, we found that 3 out of 33 cases had extra-cerebral malformations. Since these cerebral and extra-cerebral malformations play a serious role in the prognosis, they should be mentioned during counselling.

It is difficult to estimate the exact prognosis because of the limited data due to the relatively low incidence of this disorder. In the literature, there is great variability among the studies evaluated. Some assessed ACC cases with additional cerebral malformations; while the others assessed ACC cases with extra-cerebral malformations. It is well known that associated cerebral and extra-cerebral abnormalities result in poor prognosis (1). The sonographic finding of ACC should be a guide for the clinician for further assessment of not only the subtle cerebral anomalies but also the additional extra-cerebral abnormalities. It is also known that $5-20 \%$ of patients who were thought to have isolated ACC in antenatal imaging were found to have associated anomalies postnatally (6). In this perspective, foetal MRI plays a critical role in detecting them (1). If available, magnetic resonance imaging should be performed since it allows direct visualisation of $\mathrm{CC}$. It can reduce false-positive rates on ultrasound while confirming $\mathrm{ACC}$, and it can assess whether this is complete or partial. Beside these advantages it can also help to detect coexisting brain abnormalities not seen on ultrasound (8). In our study, 5 out of 33 of patients had foetal MRI to confirm the sonographic diagnosis of ACC; overall, 4 out of $5(80 \%)$ had additional cerebral malformations, including hydrocephalus and ventriculomegaly, while one had isolated ACC on foetal MRI. 
Table 1. Patient characteristics

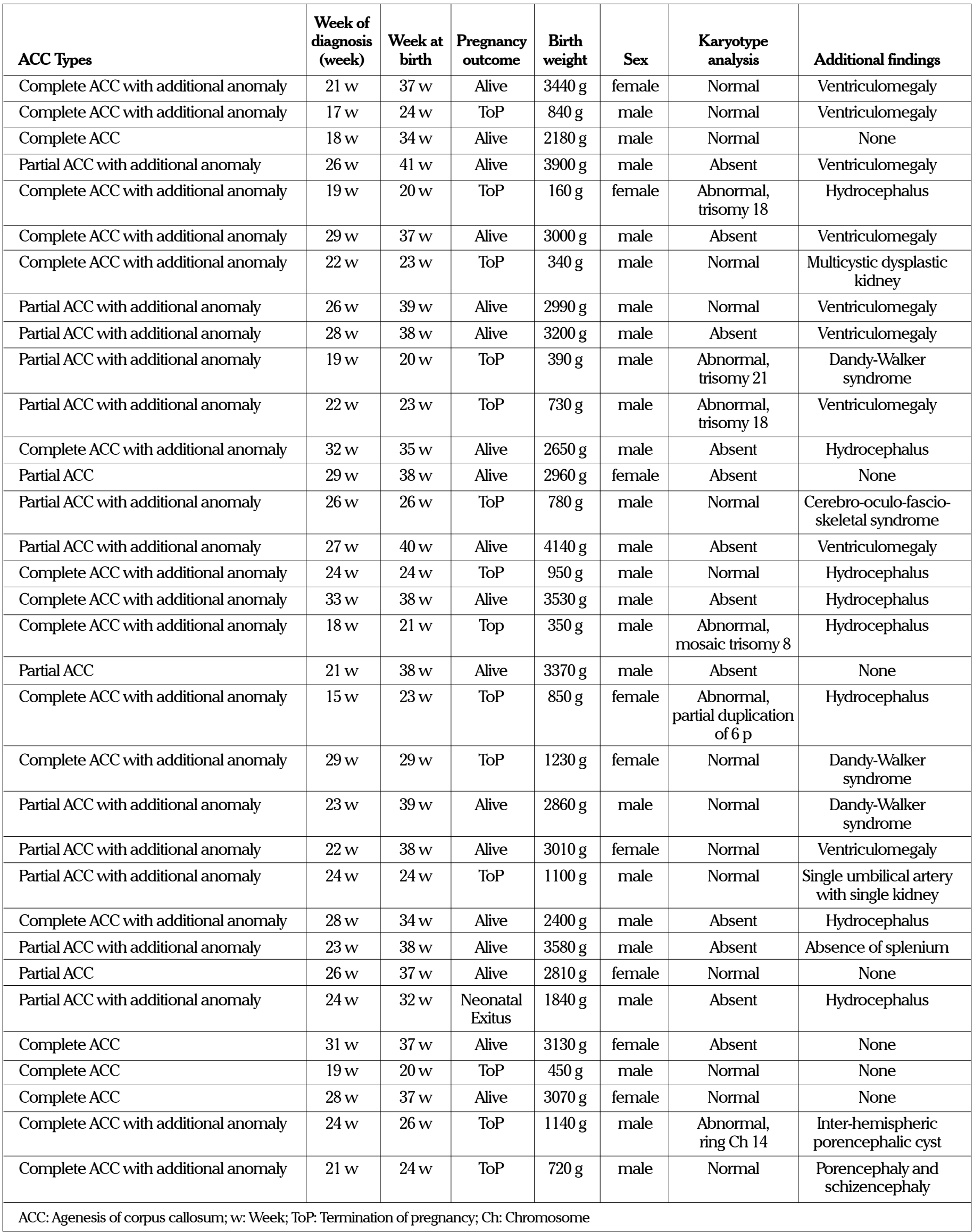


Moutard et al. (9) reported that during postnatal neuropsychological follow-up, the median intellectual quotient (IQ) was within the normal range, regardless of whether ACC is complete or partial. Since distinguishing these two by foetal ultrasonography is difficult, the clinician should pay more attention to associated cerebral and extra-cerebral anomalies.

In conclusion, the diagnosis of a congenital brain malformation creates great anxiety among the parents. In our clinical practice, since findings of additional cerebral and extra-cerebral abnormalities have a considerable effect on prognosis, we performed detailed examination with genetic counselling. Although a male preponderance was observed in our study, a gender-based research protocol is not advisable. In isolated cases of ACC, long-term neurodevelopmental outcome is expected to be normal in approximately $75 \%$ of cases, meaning that continuation of pregnancy can be preferred (5). On the other hand, if the case is not an isolated one, to the best of our knowledge, termination should be offered because of poor neurodevelopmental prognosis. For an exact diagnosis, the evaluation of CC should be postponed until after 20 weeks of gestation and foetal MRI should be offered in cases of uncertainty about termination versus continuation of pregnancy. In continuing pregnancies, delivery should be performed in multidisciplinary centres for the benefit of the infant.

Ethics Committee Approval: Ethics committee approval was not obtained because this study was designed as a retrospective case series study.

Informed Consent: Written informed consent was obtained from patients who participated in this study.

Peer-review: Externally peer-reviewed.

Author contributions: All authors contributed equally during the preparation of this manuscript.
Conflict of Interest: No conflict of interest was declared by the authors.

Financial Disclosure: The authors declared that this study has received no financial support.

\section{References}

1. Vasudevan C, McKechnie L, Levene M. Long-termoutcome of antenatally diagnosed agenesis of corpus callosum and cerebellar malformations. Semin Fetal Neonatal 2012; 17: 295-300. [CrossRef]

2. Volpe P, Paladini D, Resta M, Stanziano A, Salvatore M, Quarantelli $\mathrm{M}$, et al. Characteristics, associations and outcome of partial agenesis of the corpus callosum in the fetus. Ultrasound Obstet Gynecol 2006; 27: 509-16. [CrossRef]

3. Comstock CH, Culp D, Gonzalez J, Boal DB. Agenesis of the corpus callosum in the fetus: its evolution and significance. J Ultrasound Med 1985; 4: 613-6.

4. Sotiriadis A, Makrydimas G. Neurodevelopment after prenatal diagnosis of isolated agenesis of the corpus callosum: an integrative review. Am J Obstet Gynecol 2012; 206: 337.1-5

5. Pilu G, Sandri F, Perolo A, Pittalis MC, Grisolia G, Cocchi G, et al. Sonography of fetal agenesis of the corpus callosum: a survey of 35 cases. Ultrasound Obstet Gynecol 1993; 3: 318-29. [CrossRef]

6. Mangione R, Fries N, Godard P, Capron C, Mirlesse V, Lacombe D, Duyme M. Neurodevelopmental outcome following prenatal diagnosis of an isolated anomaly of the corpus callosum. Ultrasound Obstet Gynaecol 2011; 37: 290-5. [CrossRef]

7. Bedeschi MF, Bonaglia MC, Grasso R, Pellegri A, Garghentino RR, Battaglia MA, et al. Agenesis of corpus callosum: clinical and genetic study in 63 young patients. Pediatr Neurol 2006; 34: 186-93. [CrossRef]

8. Santo S, D'Antonio F, Homfray T, Rich P, Pilu G, Bhide A, Thilaganathan B, Papageorghiou AT. Counseling in fetal medicine: agenesis of the corpus callosum. Ultrasound Obstet Gynecol 2012; 40: 513-21. [CrossRef]

9. Moutard ML, Kieffer V, Feingold J, Kieffer F, Lewin F, Adamsbaum $\mathrm{C}$, et al. Agenesis of corpus callosum: prenatal diagnosis and prognosis. Childs Nerv Syst 2003; 19: 471-6. [CrossRef] 